\title{
Study of class I integron in a Burkholderia cepacia complex strain isolated from blood colture
}

\author{
Linda Furlanis, Lucia Corich, Lucilla Dolzani, Enrico Angelo Tonin, Cristina Lagatolla \\ Dipartimento di Scienze della Vita, Università degli Studi di Trieste
}

Key words: Burkholderia cepacia complex (Bcc), Antibiotic resistence, Class I integrons

Studio di un integrone di classe $I$ in un ceppo di Burkholderia cepacia isolato da emocoltura

\section{SUMMARY}

The Burkholderia cepacia complex $(\mathrm{Bcc})$ consists of several species that cause lung infections in patients with cystic fibrosis but are also capable to colonize immunocompromised patients. Once established, the infection is usually difficult to eradicate, as Bcc is intrinsically resistant to many antibiotics. Besides, the acquisition of additional resistance determinants by horizontal gene transfer makes very difficult the therapeutic approach to these infections. Among horizontally acquired DNAs, integrons have been frequently reported in many Gramnegative bacteria that affect human health, but they have not been found frequently in Burkholderia isolates until now.

In the present work we report on a Bcc isolate, recovered from the blood of an immunocompromised patient, that carries a $2.3 \mathrm{~kb}$ class I integron already described in a Salmonella enterica isolate eight years ago, coding for aacA4, aadAI and catB2 in its cassette array.

\section{INTRODUZIONE}

Il complesso della Burkholderia cepacia (Bcc) comprende diverse specie, note soprattutto perché responsabili di gravi infezioni polmonari in pazienti affetti da fibrosi cistica (FC).

Questi microorganismi però sono anche in grado di causare infezioni nosocomiali in pazienti immunocompromessi, grazie alla loro capacità di colonizzare forniture di acqua, disinfettanti e strumenti utilizzati per la dialisi $(3,4,5)$.

Una volta instaurata, l'infezione risulta difficile da eradicare, poiché il batterio è intrinsecamente resistente a numerosi antibiotici.

Inoltre, anche in Bcc, i meccanismi di resistenza si stanno diffondendo mediante integroni, elementi genetici che favoriscono l'acquisizione di "cassette geniche" codificanti determinanti di resistenza.

\section{METODI}

Dall'emocoltura di una paziente di 83 anni ricoverata presso un reparto di cardiologia (unità coronarica) è stato isolato un ceppo di Bcc.

L'identificazione e l'antibiogramma (Tabella 1) sono stati eseguiti con Vitek 2 e l'identificazione è stata confermata con API20NE.

La presenza di integroni di classe I è stata investigata mediante PCR con primer omologhi alle regioni costanti (2), seguita da clonazione e sequenziamento del frammento amplificato. Per dimostrare la collocazione dell'integrone, il DNA plasmidico è stato estratto mediante lisi alcalina e analizzato tramite elettroforesi in campo pulsato (PFGE).

\section{RISULTATI}

L'amplificazione della regione variabile dell'integrone ha prodotto un frammento di $2.3 \mathrm{~Kb}$, che è stato clonato in un vettore plasmidico e interamente sequenziato.

Tabella I. Valutazione della sensibilità agli antibiotici del ceppo BTS29.

\begin{tabular}{|c|c|c|}
\hline \multirow{2}{*}{$\begin{array}{c}\text { ANTIBIOTICO } \\
\text { Amikacina } \\
\end{array}$} & \multicolumn{2}{|c|}{ MIC ( $\mu \mathrm{G} / \mathrm{mL})$} \\
\hline & $>=64$ & $\mathbf{R}$ \\
\hline Amoxicillina/Ac. Clavulanico & $>=32$ & $\mathrm{R}$ \\
\hline Ampicillina & $>=32$ & $\mathrm{R}$ \\
\hline Cefazolina & $>=64$ & $\mathrm{R}$ \\
\hline Cefepime & 4 & $\mathrm{~S}$ \\
\hline Cefotaxime & $>=64$ & $\mathrm{R}$ \\
\hline Cefoxitina & $>=64$ & $\mathrm{R}$ \\
\hline Ceftazidime & 4 & $\mathrm{~S}$ \\
\hline Ciprofloxacina & $\mathrm{I}$ & $\mathrm{S}$ \\
\hline Cloramfenicolo & $>=32$ & $\mathbf{R}$ \\
\hline Gentamicina & $>=16$ & $\mathbf{R}$ \\
\hline Imipenem & 8 & $\mathrm{R}$ \\
\hline Netilmicina & $>=32$ & $\mathbf{R}$ \\
\hline Nitrofurantoina & $>=512$ & $\mathrm{R}$ \\
\hline Norfloxacina & 2 & $\mathrm{~S}$ \\
\hline Piperacillina & 32 & $\mathrm{I}$ \\
\hline Piperacillina/Tazobactam & $<=4$ & $\mathrm{~S}$ \\
\hline Tetraciclina & $>=16$ & $\mathrm{R}$ \\
\hline Trimetoprim/Sulfametossazolo & 40 & $\mathrm{~S}$ \\
\hline
\end{tabular}

\section{Corresponding author: Linda Furlanis}

Dipartimento di Scienze della Vita, Università di Trieste

Via L. Giorgieri I - 34I00 Trieste, Italia - Tel.: 040-5583693 - Fax: 040-558369|

E-mail: Ifurlanis@units.it 


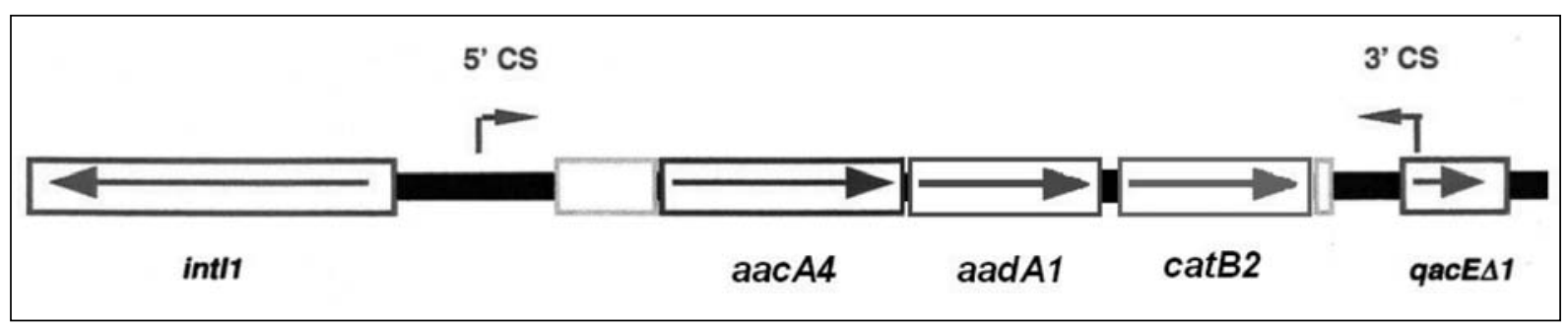

Figura I. Rappresentazione dell'integrone identificato in BTS29.

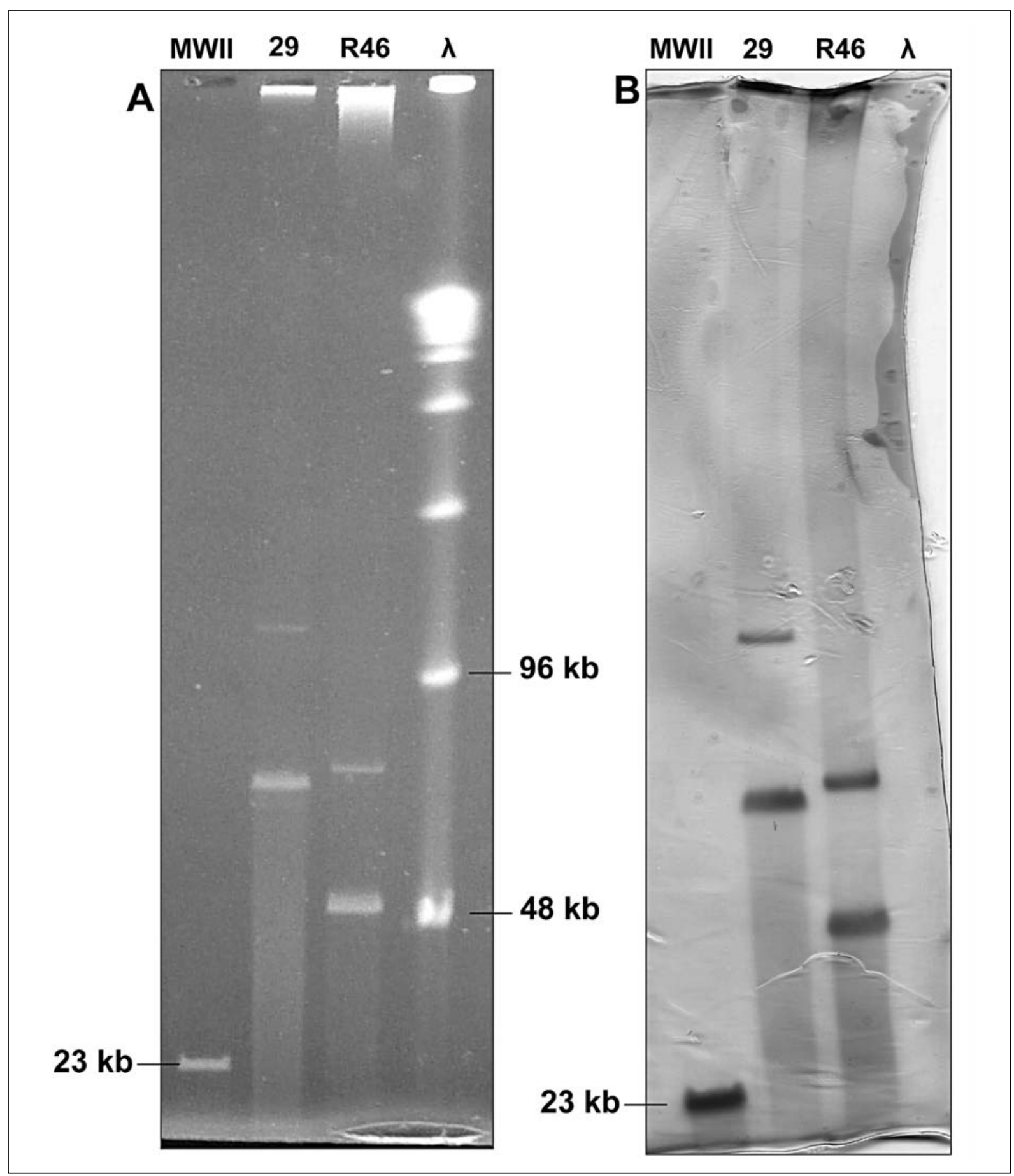

Figura II. Elettroforesi e ibridazione su gel (I) con sonda omologa al gene Intl.

A. Separazione mediante PFGE in gel di agarosio all'।\% dei plasmidi estratti dal ceppo BTS29.

B. Ibridazione su gel con sonda omologa al gene Int l marcata con digossigenina. MW: DNA Molecular Weight Marker II marcato con digossigenina. 
La regione variabile è risultata costituita da tre cassette geniche, aacA4, aadA1, catB2 (Figura I), le prime due conferenti resistenza ad aminoglicosidi, la terza al cloramfenicolo. Lo stesso integrone era stato ritrovato in precedenza solamente in una Salmonella enterica Enteritidis isolata a Roma (6). Inoltre il gene aacA4 presenta una forma allelica mutata, precedentemente identificata in isolati Bcc da pazienti FC della nostra regione. L'elettroforesi in campo pulsato (PFGE) del DNA plasmidico seguito dall'ibridazione su gel con la sonda omologa al gene dell'integrasi di classe I (intI1) ha dimostrato che questo integrone si trova in un plasmide di circa $70-80 \mathrm{~Kb}$ (Figura II).

\section{CONCLUSIONI}

Questi risultati suggeriscono che la circolazione degli integroni all'interno del Bcc sia comparabile a quella riscontrata in altre specie batteriche, nonostante le citazioni in letteratura fino a questo momento non siano state molto numerose. Riteniamo che l'acquisizione di determinanti della resistenza in un genere batterico che presenta già numerose resistenze intrinseche, ulteriormente aggravata dal fatto che tali determinanti sono vei- colati da plasmidi e quindi potenzialmente trasmissibili, sia un fatto estremamente preoccupante.

\section{BIBLIOGRAFIA}

1. Khan SA, Nawaz MS, Khan AA, Cerniglia CE. Direct in-gel hybridization of digoxigenin-labelled non-radioactive probes. Mol Cell Probes 1999; 13(3): 233-7.

2. Lévesque C, Piché L, Larose C, Roy PH. PCR mapping of integrons reveals several novel combinations of resistance genes. Antimicrob Agents Ch 1995; 39(1): 185-91.

3. Lo Cascio G, Bonora MG, Zorzi A, et al. A napkinassociated outbreak of Burkholderia cenocepacia bacteraemia in haemodialysis patients. J Hosp Infect 2006; 64(1): 56-62.

4. Romero-Gómez MP, Quiles-Melero MI, Peña García $\mathrm{P}$, et al. Outbreak of Burkholderia cepacia bacteremia caused by contaminated chlorhexidine in a hemodialysis unit. Infect Control Hosp Epidemiol 2008; 29(4): 377-8.

5. Souza AV, Moreira CR, Pasternak J, et al. Characterizing uncommon Burkholderia cepacia complex isolates from an outbreak in a haemodialysis unit. J Med Microbiol 2004; 53(10): 999-1005.

6. Villa L, Mammina C, Miriagou V, et al. Multidrug and broad-spectrum cephalosporin resistance among Salmonella enterica serotype Enteritidis clinical isolates in southern Italy. J Clin Microbiol 2002; 40(7): 2662-5. 